\title{
Research on a Path-Tracking Control System for Articulated Tracked Vehicles
}

\author{
Da Cui - Guoqiang Wang - Huanyu Zhao - Shuai Wang* \\ Jilin University, School of Mechanical and Aerospace Engineering, China
}

To achieve path-tracking control of articulated tracked vehicles (ATVs), a control system is designed according to the structural characteristics of the ATVs. The distance deviation and heading angle deviation between the vehicle and the pre-set trajectory are employed as input variables to the controller to control the relative deflection angle of the hinge of the vehicle and the velocity of the sprockets and realize the function of the path tracking control. The effects of two control algorithms are simulated with a RecurDyn/Simulink co-simulation; the results show that the fuzzy proportional-integral-derivative (PID) has a better control effect and precision when compared with the PID method. To verify the effect of the proposed controller, a scaled model test platform that is based on visual navigation is developed. Physical experiments were conducted to show the efficacy of the proposed path-tracking control system, which can effectively track the pre-set trajectory and achieve an optimal control outcome.

Keywords: articulated tracked vehicle, path tracking, fuzzy PID controller, co-simulation, visual navigation

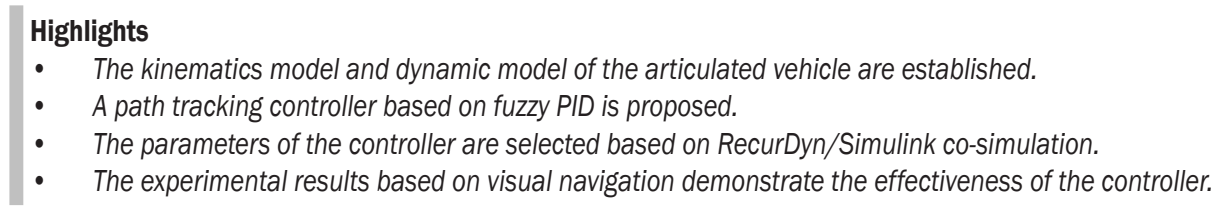

\section{INTRODUCTION}

An articulated tracked vehicle (ATV) is composed of two groups of double-track vehicle units that are connected in series by an articulated structure. Different from four-track [1] and six-track steering vehicles [2], this vehicle is driven by actuators, such as hydraulic or electric cylinders, which produce a relative deflection of the front and rear around the hinge point to enable steering [3]. Compared with traditional two-track vehicles, ATVs have low ground pressure and excellent mobility. Due to these advantages, ATVs have been employed in various applications, in the military, agriculture, forestry, and elsewhere [4]. For example, Fig. 1 shows a typical ATV that is employed for military purposes. Complex and hazardous working environments require autonomous or semi-autonomous navigation systems to minimize the exposure of people to risk. Therefore, research on the automatic motion control system for ATVs is imperative.

The motion of an ATV is a complex problem that has attracted considerable attention. Based on the steady-state and unsteady-state plane steering performance of a single-tracked vehicle, Watanabe and Kitano [5] established a mathematical model for plane steering of an ATV, including the vehicle and ground characteristics. Alhimdani [6] theoretically analysed the steering of the wagon-type articulation of tracked vehicles and indicated the effects of the articulated structure and geometric dimensions of trailers on the steering process. Sasaki et al. [7] introduced the design, structural, and driving characteristics of a four-degree-of-freedom articulated tracked vehicle. This ATV can reduce the ground pressure distribution by actively controlling the relative postures of the vehicle's front and rear segments.

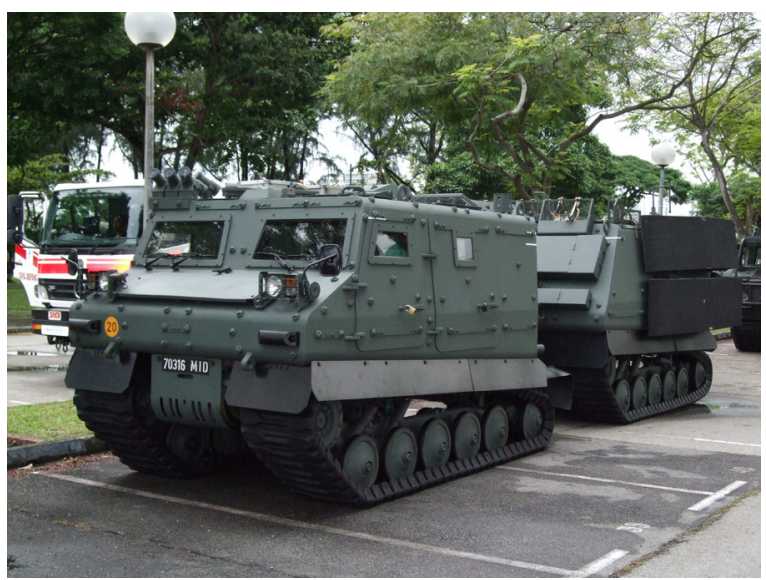

Fig. 1. Typical articulated tracked vehicle

Recently, increasing attention has been given to research on the motion of an electric ATV. Fijalkowski [8] proposed an all-electric intelligent ATV, which has better performance on soft ground, to improve 
the mobility and steerability of traditional vehicles. Zhao et al. [4] established a virtual prototype model of an ATV using multibody dynamics software to investigate the steady-state steering performance for the condition of changing the track speed difference on both sides, theoretical steering radius, and ground friction coefficient. Moreover, the electromechanical coupling model of an ATV is established in [9].

Similar to the automatic navigation or trajectory tracking of mobile robots [10] and [11] and wheeled vehicles [12] to [18], the automatic motion control of tracked vehicles has become a focus of research interest. Hong et al. [19] designed a path-tracking study of submarine crawler mining robots according to the skid dynamics model and the interaction between the track and the ground. Wang et al. [20] designed a tracked vehicle remote control system by using the internet and machine vision and compared the path-tracking control effects of various intelligent control algorithms. Zou et al. [21] established a dynamic model of tracked vehicles that considers track slippage and presents a modified proportionalintegral-derivative (PID) computed-torque controller. The numerical simulation results show high accuracy of the motion control. To improve the control accuracy of a tracked vehicle, Zhou et al. [22] proposed a model predictive control algorithm by using a linear kinematics model, which shows feasible results both in a simulation environment and experiments. In addition to traditional double-track vehicles, Wang et al. [23] designed an autonomous navigation system of a six-crawler machine that is based on visual tracking control technology.

However, research about the automatic motion control of ATVs is lacking. This study aims to design an optimal path tracking control system for ATVs. Among the diverse types of control methodologies, classical PID controllers have gained wide acceptance due to their advantages of simple structure, ease of design and low cost in implementation [24]. These features render PID control preferable to other nonlinear control methods in various control applications [25]. For example, the design of an adaptive controller involves the determination of a couple of adaptive adjusting terms, which cause design complexity [21]. The implementation of model predictive control (MPC) requires solving an optimization at each step, which requires a large amount of computation [26].

PID control is capable of stabilizing nonlinear systems, whose performance has been compared with a linear quadratic regulator (LQR) for wheeled

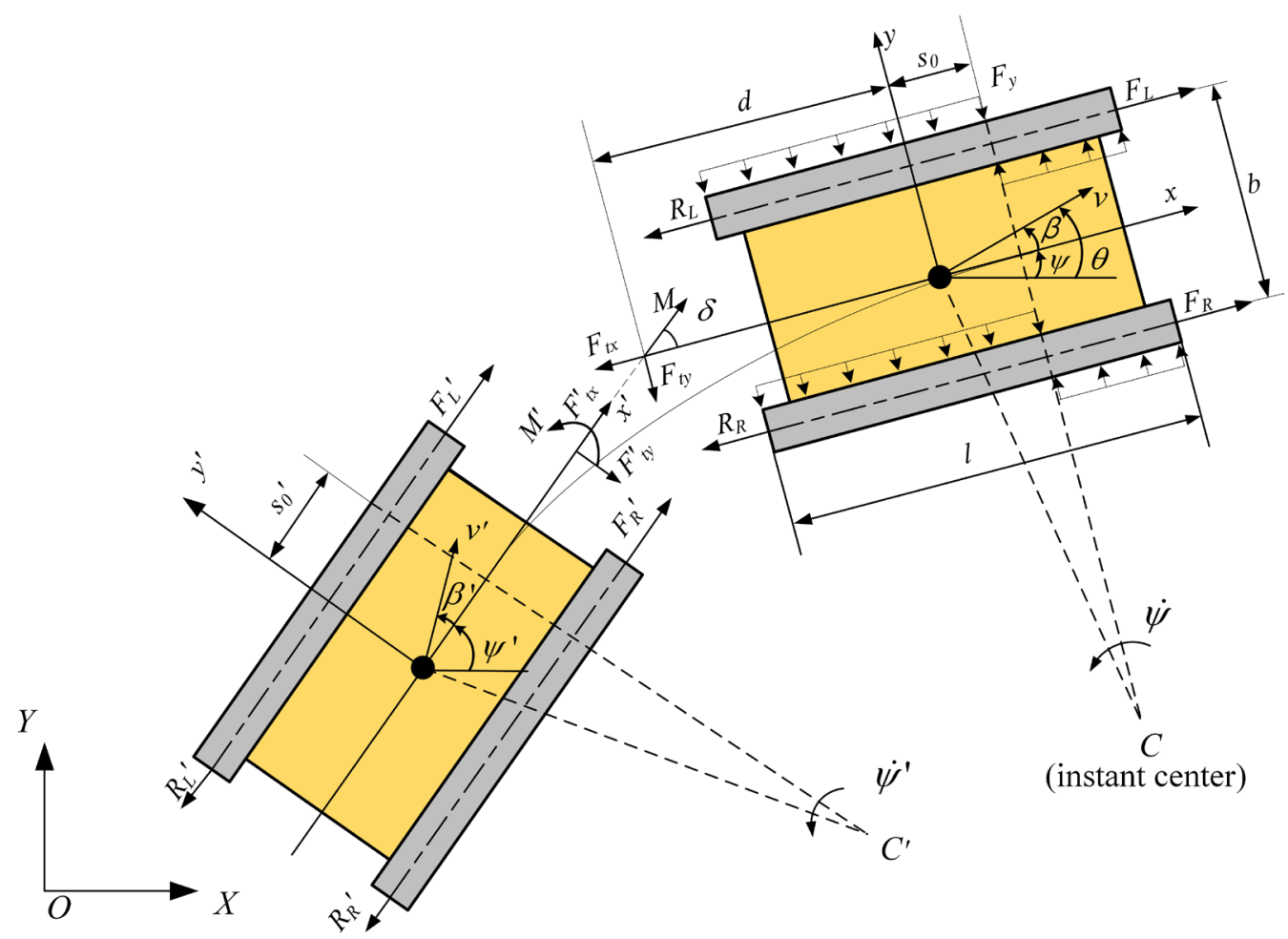

Fig. 2. Steering movement diagram of an ATV 
robots [27] and linear quadratic Gaussian (LQG) control for autonomous vehicles [28]. Both these controllers satisfy the operation condition, but the PID controller shows ease of design and implementation. However, with a classical PID controller, it is difficult to achieve optimal control performance for nonlinear and complex systems. In order to achieve the desired performance, fuzzy logic control is particularly appropriate to enhance them due to its ability to translate the operator's intelligence to automatic control [29] and [30].

In this paper, we proposed a path-tracking control system for ATVs based on fuzzy PID control and visual navigation. This study analyses the steering characteristics of ATV, and the dynamic model of an ATV is introduced. Considering the distance and heading angle deviations between the vehicle and the pre-set path as the input variables and the deflection angle of the hinge point and the speed of the driving sprockets as the outputs of the controller, an ATV pathtracking control system is developed based on fuzzy PID control algorithm. The control system is being tested by a co-simulation in RecurDyn/Simulink. An experimental platform for path tracking control is created to verify the effect in practical applications. The experimental platform uses a camera to obtain the deviation, which is processed as controller input. The experimental results show agreement with the simulation results, indicating that the designed controller can effectively track the pre-set trajectory.

\section{DYNAMIC MODELLING}

\subsection{Kinematics}

An ATV consists of two identical vehicles, a front vehicle and a rear vehicle, linked with a rigid articulated joint. The articulated steering is being performed on the middle joint, by changing the corresponding articulated angle between the front vehicle and the rear vehicle as it is being indicated in Fig. 2. The articulating device is driven by actuators, such as hydraulic or electric cylinders, which push the front and rear vehicles to deflect around the articulated joint at the same time. Compared with the skid steering, articulated steering requires less power.

The steering motion of the vehicle in the inertial coordinate system $X O Y$ is shown in Fig. 2, where xoy and $x^{\prime} o^{\prime} y^{\prime}$ are vehicle coordinate systems fixed at the centre of the body of the front vehicle and the rear vehicle, respectively. Assume that the parameters of the front and rear vehicle bodies are identical. In the following expressions, $m$ is the mass of the single vehicle, $l$ is the grounding length of the crawler, $b$ is the distance of the centre between the two crawlers, $d$ is the distance between the articulated steering centre and the centre of the front and rear vehicle, and $I_{Z}$ is the moment of inertia of the single vehicle. When the deflection angle between the front vehicle and the rear vehicle is $\delta$, the motion relationship between them can be expressed as:

$$
\begin{aligned}
& \psi^{\prime}=\psi+\delta, \\
& \dot{x}^{\prime}=\dot{x} \cos \delta+(\dot{y}-d \dot{\psi}) \sin \delta, \\
& \dot{y}^{\prime}+d \dot{\psi}^{\prime}=-\dot{x} \sin \delta+(\dot{y}-d \dot{\psi}) \cos \delta,
\end{aligned}
$$

where $\dot{x}$ and $\dot{y}$ are components of the total velocity $v$ of the front vehicle combination, $\dot{x}^{\prime}$ and $\dot{y}^{\prime}$ are components of the total velocity $v^{\prime}$ of the rear vehicle combination, $\psi$ and $\psi^{\prime}$ are the heading angle of the front vehicle and the rear vehicle, respectively. The velocities $v$ and $v^{\prime}$ are considered to have the same changes with respect to the deflection angle velocity $\dot{\delta}$ of the articulated joint, indicated by $\psi$ and $\psi^{\prime}$.

During steering, the deviation between the actual speed direction of the vehicle and the longitudinal direction of the vehicle body is the sideslip angle, represented as $\beta$ and $\beta^{\prime}$, which can be calculated by:

$$
\beta=\arctan (\dot{y} / \dot{x}), \quad \beta^{\prime}=\arctan \left(\dot{y}^{\prime} / \dot{x}^{\prime}\right) .
$$

By defining the state space vector as $\xi=[\mathrm{XY} \psi \delta]^{\mathrm{T}}$, the complete kinematics expression based on the front vehicle can be given as:

$$
\left[\begin{array}{c}
\dot{X} \\
\dot{Y} \\
\dot{\psi} \\
\dot{\delta}
\end{array}\right]=\left[\begin{array}{cc}
\mathrm{c}(\psi+\beta) & 0 \\
\mathrm{~s}(\psi+\beta) & 0 \\
\frac{\mathrm{s} \gamma \mathrm{c} \delta-\mathrm{c} \gamma \mathrm{s} \delta}{l\left[\mathrm{c} \beta^{\prime}+\mathrm{c} \lambda\right]} & \frac{-\mathrm{c} \beta^{\prime}}{\mathrm{c} \beta^{\prime}+\mathrm{c} \lambda} \\
0 & 1
\end{array}\right]\left[\begin{array}{l}
v \\
\dot{\delta}
\end{array}\right]
$$

where $X$ and $Y$ denote the position of the centre of mass of the front vehicle in the inertial coordinate system. Define $\gamma=\beta-\beta^{\prime}$ and $\lambda=\delta-\beta^{\prime}, \mathrm{c}(\cdot)$ and $\mathrm{s}(\cdot)$ stand for $\cos (\cdot)$ and $\sin (\cdot)$, respectively.

The track slips for each track can be calculated with the following formula [19]:

$$
\begin{gathered}
i_{L}=1-\frac{v_{L}}{r \omega_{L}}=1-\frac{\dot{x}+(b / 2) \dot{\psi}}{r \omega_{L}}, \\
i_{R}=1-\frac{v_{R}}{r \omega_{R}}=1-\frac{\dot{x}-(b / 2) \dot{\psi}}{r \omega_{R}}, \\
i_{L}{ }^{\prime}=1-\frac{v_{L}{ }^{\prime}}{r \omega_{L}{ }^{\prime}}=1-\frac{\dot{x}+(b / 2) \dot{\psi}^{\prime}}{r \omega_{L}{ }^{\prime}}, \\
i_{R}{ }^{\prime}=1-\frac{v_{R}{ }^{\prime}}{r \omega_{R}{ }^{\prime}}=1-\frac{\dot{x}-(b / 2) \dot{\psi}^{\prime}}{r \omega_{R}{ }^{\prime}},
\end{gathered}
$$


where $\omega_{L}, \omega_{R}, \omega_{L}{ }^{\prime}$ and $\omega_{R}{ }^{\prime}$ are the sprocket velocities of each track, $r$ is the pitch radius of the sprocket, and $v_{L}, v_{R}, v_{L}{ }^{\prime}$ and $v_{R}{ }^{\prime}$ are the actual velocities of each track.

\subsection{Dynamic Model}

As shown in Fig. 2, $F_{L}, F_{R}, F_{L}{ }^{\prime}$ and $F_{R}{ }^{\prime}$ denote the tractive forces of the tracks, $R_{L}, R_{R}, R_{L}{ }^{\prime}$ and $R_{R}{ }^{\prime}$ denote the longitudinal resistance $F_{t x}, F_{t v}, F_{t x}{ }^{\prime}$ and $F_{t v}{ }^{\prime}$ are the forces that act on the articulation joint, and $M_{T}$ and $M_{T}{ }^{\prime}$ are the steering torque. The nonlinear dynamic model of the ATV can be expressed as follows [5]:

- For the front vehicle:

$$
\begin{aligned}
m \ddot{x} & =m \dot{y} \dot{\psi}+\left(F_{L}+F_{R}\right)-\left(R_{L}+R_{R}\right)-F_{t x}, \\
m \ddot{y} & =-m \dot{x} \dot{\psi}+F_{y}-F_{t y}, \\
I_{z} \ddot{\psi} & =M_{L}-M_{R}-F_{t y} d+M_{t} .
\end{aligned}
$$

- For the rear vehicle:

$$
\begin{aligned}
m^{\prime} \ddot{x}^{\prime} & =m \dot{y}^{\prime} \dot{\psi}^{\prime}+\left(F_{l}{ }^{\prime}+F_{r}{ }^{\prime}\right)-\left(R_{l}{ }^{\prime}+R_{r}{ }^{\prime}\right)+F_{t x}{ }^{\prime}, \\
m^{\prime} \ddot{y}^{\prime} & =-m \dot{x}^{\prime} \dot{\psi}^{\prime}+F_{y}{ }^{\prime}-F_{t y}{ }^{\prime}, \\
I_{z} \ddot{\psi}^{\prime} & =M_{l}{ }^{\prime}-M_{r}{ }^{\prime}+F_{t y}{ }^{\prime} d-M_{t}{ }^{\prime} .
\end{aligned}
$$

Detailed expressions of the dynamic model are presented as follows.

\subsubsection{Tractive Force}

The maximum tractive force produced by a track is determined by the contact area $A$ between the track and terrain and the maximum shear strength of the terrain $\tau_{\max }[31]$ :

$$
F_{\max }=A \tau_{\max }=A(c+p \tan \phi),
$$

where $c$ is the apparent cohesion, $\phi$ is the angle of internal shearing resistance of the terrain, and $p$ is the normal pressure. The load is assumed to be uniformly distributed over the contact area.

When the track slip is $i$, the tractive force of a track can be calculated with [32]:

$$
F=F_{\max }\left[1-\frac{K}{i l}\left(1-e^{-i l / K}\right)\right],
$$

where $K$ is the soil shear deformation modulus.

\subsubsection{Resistance Forces}

The longitudinal resistance force is created by sinkage and rut formation. When driving on hard pavement, the value is relatively small compared with the tractive force. The longitudinal resistance force can be expressed as:

$$
\begin{gathered}
R_{L}=R_{R}=\mu_{f} \frac{W}{2}, \\
R_{L}{ }^{\prime}=R_{R}{ }^{\prime}=\mu_{f} \frac{W^{\prime}}{2},
\end{gathered}
$$

where $\mu_{f}$ is the coefficient of longitudinal motion resistance and $W=W^{\prime}=m g$ are the normal load on the track.

The lateral resistance forces $F_{v}$ and $F_{v}{ }^{\prime}$ can be calculated by:

$$
\begin{aligned}
F_{y} & =2 \operatorname{sgn}(\dot{\psi}) \mu_{t}\left[\int_{-l / 2}^{s_{0}} \frac{W}{2 l} d x-\int_{s_{0}}^{l / 2} \frac{W}{2 l} d x\right] \\
& =2 \operatorname{sgn}(\dot{\psi}) \mu_{t} s_{0} \frac{W}{l}, \\
F_{y}^{\prime} & =2 \operatorname{sgn}\left(\dot{\psi}^{\prime}\right) \mu_{t}\left[\int_{-l^{\prime} / 2}^{s^{\prime}} \frac{W^{\prime}}{2 l^{\prime}} d x-\int_{s_{0}^{\prime}}^{l^{\prime} / 2} \frac{W^{\prime}}{2 l^{\prime}} d x\right] \\
& =2 \operatorname{sgn}\left(\dot{\psi}^{\prime}\right) \mu_{t} s_{0}{ }^{\prime} \frac{W^{\prime}}{l^{\prime}},
\end{aligned}
$$

where $\mu_{t}$ is the motion resistance coefficient in the lateral direction.

\subsubsection{Moment of the Longitudinal Forces and Lateral Forces}

The steering moments caused by the longitudinal forces that act on the front and rear tracks with respect to their respective centroids can be expressed as follows:

- For the front vehicle:

$$
M_{l}=\left(F_{L}-R_{L}\right) \frac{b}{2}-\left(F_{R}-R_{R}\right) \frac{b}{2}=\left(F_{L}-F_{R}\right) \frac{b}{2} .
$$

- For the rear vehicle:

$$
M_{l}^{\prime}=\left(F_{L}{ }^{\prime}-R_{L}{ }^{\prime}\right) \frac{b}{2}-\left(F_{R}{ }^{\prime}-R_{R}{ }^{\prime}\right) \frac{b}{2}=\left(F_{L}{ }^{\prime}-F_{R}{ }^{\prime}\right) \frac{b}{2} \text {. }
$$

The moments of the lateral forces are given by:

$$
\begin{aligned}
M_{r} & =2 \operatorname{sgn}(\dot{\psi}) \mu_{t}\left[\int_{-l / 2}^{s_{0}} \frac{W}{2 l} x d x-\int_{s_{0}}^{l / 2} \frac{W}{2 l} x d x\right] \\
& =\operatorname{sgn}(\dot{\psi}) \mu_{t} \frac{W}{l}\left(\frac{l^{2}}{4}-s_{o}^{2}\right), \\
M_{r}{ }^{\prime} & =2 \operatorname{sgn}\left(\dot{\psi}^{\prime}\right) \mu_{t}\left[\int_{-l^{\prime} / 2}^{s_{0^{\prime}}} \frac{W^{\prime}}{2 l^{\prime}} x d x-\int_{s_{0}^{\prime}}^{l^{\prime} / 2} \frac{W^{\prime}}{2 l^{\prime}} x d x\right] \\
& =\operatorname{sgn}(\dot{\psi}) \mu_{t} \frac{W}{l^{\prime}}\left(\frac{l^{\prime 2}}{4}-s_{0}{ }^{2}\right) .
\end{aligned}
$$




\subsubsection{Interaction Force between Front and Rear Vehicles}

As mentioned above, steering of the articulated vehicle is accomplished by relative turns in the front-rear vehicle plane. The steering torques of the front and rear vehicles actuated by the articulating device are equal and opposite directions [5]. For the decomposition of the forces on the articulated joint, the balance is expressed as follows:

$$
\left[\begin{array}{l}
F_{t x} \\
F_{t y} \\
M_{t}
\end{array}\right]=\left[\begin{array}{ccc}
\mathrm{c} \delta & \mathrm{s} \delta & 0 \\
-\mathrm{s} \delta & \mathrm{c} \delta & 0 \\
0 & 0 & 1
\end{array}\right]\left[\begin{array}{l}
F_{t x}{ }^{\prime} \\
F_{t y}{ }^{\prime} \\
M_{t}{ }^{\prime}
\end{array}\right] .
$$

Substitute these formulas into Eqs. (5) and (6), a non-linear dynamics model of the ATV can be expressed in terms of $\dot{x}, \dot{y}, \dot{\psi}$ as:

$$
\begin{aligned}
& {\left[\begin{array}{ccc}
-\mathrm{m}(2+\mathrm{s} \delta \mathrm{c} \delta) & m \mathrm{~s} \delta \mathrm{c} \delta & m d \mathrm{~s} \delta \mathrm{c} \delta \\
-m \mathrm{~s}^{2} \delta & -m\left(1+\mathrm{c}^{2} \delta\right) & m d \\
-m d \mathrm{~s} \delta & m d(\mathrm{c} \delta-1) & 2 I+m d^{2} \mathrm{c} \delta
\end{array}\right]\left[\begin{array}{c}
\ddot{x} \\
\ddot{y} \\
\ddot{\psi}
\end{array}\right]} \\
& =\left[\begin{array}{c}
f(1) \mathrm{c} \delta+f(2) \mathrm{s} \delta+f(3) \\
f(1) \mathrm{s} \delta-f(2) \mathrm{c} \delta+f(4) \\
f(2)+f(4)-f(5)+f(6)+I \ddot{\delta}
\end{array}\right],
\end{aligned}
$$

where $f(1) \sim f(6)$ are expressed as:

$$
\begin{gathered}
f(1)=m[g(1)+g(2)]-\left(F_{L}{ }^{\prime}+F_{R}{ }^{\prime}\right), \\
f(2)=-m[g(3)-g(4)]+F_{y}{ }^{\prime}, \\
f(3)=m \dot{y} \dot{\psi}-\left(F_{L}+F_{R}\right), \\
f(4)=-m \dot{x} \dot{\psi}-F_{y}, \\
f(5)=\frac{b}{2}\left(F_{L}-F_{R}\right)+\frac{b}{2}\left(F_{L}{ }^{\prime}-F_{R}{ }^{\prime}\right), \\
f(6)=\frac{b}{2} g(5)+g(6),
\end{gathered}
$$

where $g(1) \sim g(6)$ are expressed as follows:

$$
\begin{gathered}
g(1)=\dot{x}(\mathrm{c} \delta-\mathrm{s} \delta) \ddot{\delta}+d \dot{\psi} \mathrm{c} \delta \cdot \dot{\delta}, \\
g(2)=\left[d \dot{\psi}^{\prime}-\dot{x} \mathrm{~s} \delta+\left(\dot{y}+d \dot{\psi}^{\prime}\right) \mathrm{c} \delta\right] \dot{\psi}, \\
g(3)=-\dot{x} \mathrm{c} \delta \cdot \dot{\delta}+d \dot{\psi}^{\prime}-(\dot{y}+d \dot{\psi}) \cdot \mathrm{s} \delta \cdot \dot{\delta}, \\
g(4)=[\dot{x} \mathrm{c} \delta+(\dot{x}+d \dot{\psi}) \mathrm{c} \delta] \dot{\psi}^{\prime}, \\
g(5)=\left(R_{L}-R_{R}\right)+\left(R_{L}{ }^{\prime}-R_{R}{ }^{\prime}\right), \\
g(6)=M_{r}+M_{r}{ }^{\prime}
\end{gathered}
$$

In this system, the control quantity is selected as $\mathbf{u}=[v, \delta]^{T}$ and the output variables are selected as $\xi_{d y n}=[X, Y, \psi, \dot{\psi}, \dot{x}, \dot{y}]^{T}$.

\subsection{Virtual Prototyping Dynamic Model}

The dynamic system of an articulated tracked vehicle is a complex non-linear system. The mathematical model is often simplified to solve the dynamic equation of its steering, while the virtual prototype simulation can yield simulation results that are closer to the real value by establishing relevant parameters that can more intuitively observe the actual driving process.

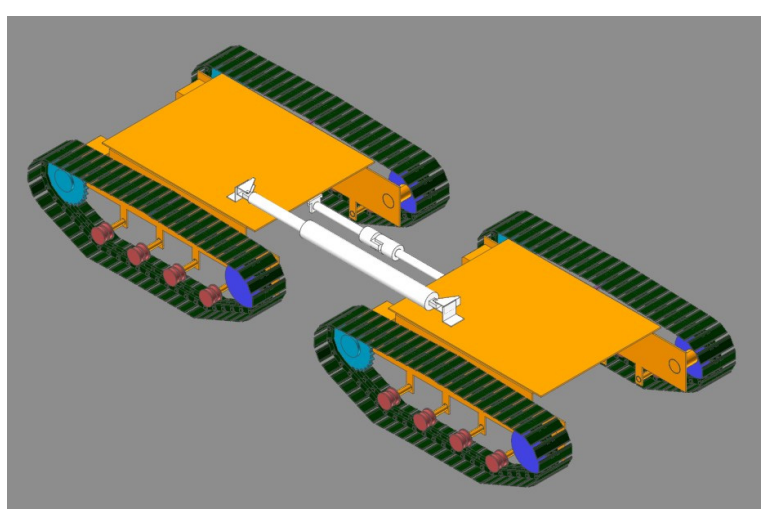

Fig. 3. Virtual prototype model

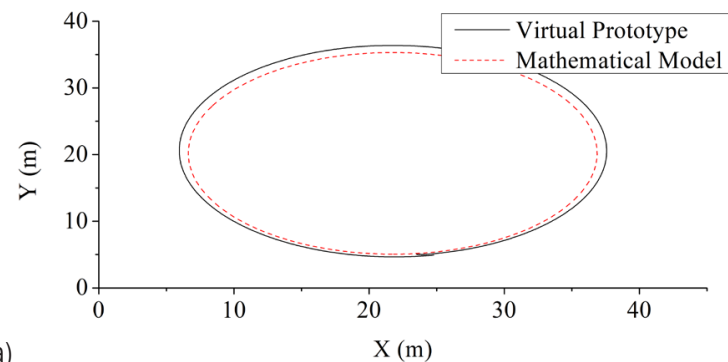

a)

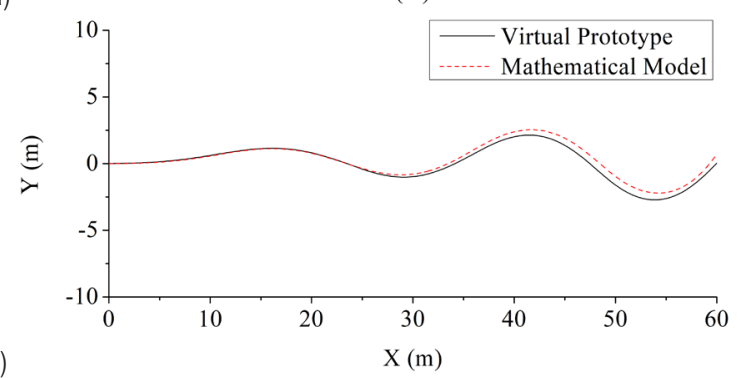

Fig. 4. Comparison of simulation results; a) steady-state steering $\left(\delta=20^{\circ}\right)$, and b) unsteady-state steering

Professional crawler modules exist in the RecurDyn multi-body dynamics software. The dynamic model of an ATV is established in RecurDyn, as shown in Fig. 3, and the control model is established in Simulink. The trajectory of the mathematical model and the virtual prototype model is compared using the same control input to verify the accuracy of the 
virtual prototype model. From the comparison of the results in Fig. 4, the change in the vehicle trajectory in both the steady-state steering process and the unsteady state steering process is consistent. This model will be employed in the simulation of the following path tracking control algorithm.

\section{CONTROLLER DESIGN}

The controller is designed to enable the ATV to track a reference path. The maximum deflection angle $\delta$ of the ATV is $20^{\circ}$, and the corresponding theoretical turning radius is $14.8 \mathrm{~m}$, in this case [4]. Since the research on research on automatic motion control of ATVs is lacking, the performance of the controller is designed with reference to the six-crawler machine [23] as its similar dynamic behaviour. The maximum percentage overshoot is set to $20 \%$, and the settling time $t_{s}$ is set to $10 e_{y, \max } / v$ for which $e_{y, \max }$ is the maximum distance deviation.

In this path-tracking problem, the reference pose $\mathbf{p}_{r}=\left[\begin{array}{lll}X_{r} & Y_{r} & \psi_{r}\end{array}\right]^{\mathrm{T}}$ and current pose $\mathbf{p}_{c}=\left[\begin{array}{lll}X & Y & \psi\end{array}\right]^{\mathrm{T}}$ are defined. The deviation between the two poses is defined as the path-tracking error, $\mathbf{e}_{p}$, and the pathtracking error based on the coordinate system of the front vehicle can be expressed as follows:

$$
\begin{aligned}
\mathbf{e}_{p} & =\left[\begin{array}{ccc}
e_{x} & e_{y} & e_{\psi}
\end{array}\right]^{T}=\mathbf{R}\left(\mathbf{p}_{r}-\mathbf{p}_{c}\right) \\
& =\left[\begin{array}{ccc}
\mathrm{c} \psi & \mathrm{s} \psi & 0 \\
-\mathrm{s} \psi & \mathrm{c} \psi & 0 \\
0 & 0 & 1
\end{array}\right]\left[\begin{array}{c}
X_{r}-X \\
Y_{r}-Y \\
\psi_{r}-\psi
\end{array}\right] .
\end{aligned}
$$

Considering the nearest point between the reference trajectory and the centre of the front vehicle as the reference point, the longitudinal tracking control deviation based on xoy-coordinate is set to zero, and the vehicle velocity is constant. The lateral error and heading angle deviation of the vehicle are considered as the control deviation and input into the controller. The control deviation is defined as follows:

$$
e_{\delta}=e_{\psi}+\arctan k \frac{e_{y}}{v} .
$$

Based on this description, the fuzzy PID controller of an ATV is proposed as:

$$
\delta(t)=K_{p} e_{\delta}+K_{i} \int_{0}^{t} e_{\delta} d t+K_{d} \frac{d e_{\delta}}{d t},
$$

where $K_{p}, K_{i}$ and $K_{d}$ are the proportional, integral and derivative gains, respectively, all positive. The proportional gain provides an overall control action proportional to the error signal through the all-pass gain factor. The functionality of integral gain is to reduce steady-state errors through lowfrequency compensation with an integrator. The functionality of derivative gain is improving transient response through high-frequency compensation by a differentiator [33].

The gains of the PID are determined online, and the corrected PID controller gains are obtained by fuzzy inference as follows [34]:

$$
\begin{aligned}
& K_{p}=\left(K_{p \max }-K_{p \text { min }}\right) K_{p}{ }^{\prime}+K_{p \text { min }}, \\
& K_{i}=\left(K_{i \text { max }}-K_{i \text { min }}\right) K_{i}{ }^{\prime}+K_{i \text { min }}, \\
& K_{d}=\left(K_{d \text { max }}-K_{d \text { min }}\right) K_{d}{ }^{\prime}+K_{d \text { min }},
\end{aligned}
$$

where $\left[K_{p \min } ; K_{p \max }\right],\left[K_{i \min } ; K_{i \max }\right]$ and $\left[K_{d \min } ; K_{d \max }\right]$ are the ranges of $K_{p}, K_{i}$ and $K_{d}$ respectively.

The procedure of finding the PID gains is called "tuning". Many PID tuning methods had been derived to determine the value of the three parameters to obtain a controller with good performance and robustness [35]. However, no tuning method thus far can replace the simple The Ziegler-Nichols (Z-N) method in terms of familiarity and ease of use to start with [33]. Tuning by experience is also a common method [21], and [34], which means that tuning through different simulation tests. In this work, the three parameters are tuned by experience with reference to the Z-N method. Furthermore, the ranges of $K_{p}, K_{i}$ and $K_{d}$ are determined based on the comparative tuning method [36]. The ranges are tuned to achieve a faster response and a smaller steady-state error compared with the well-tuned PID gains through simulation tests.

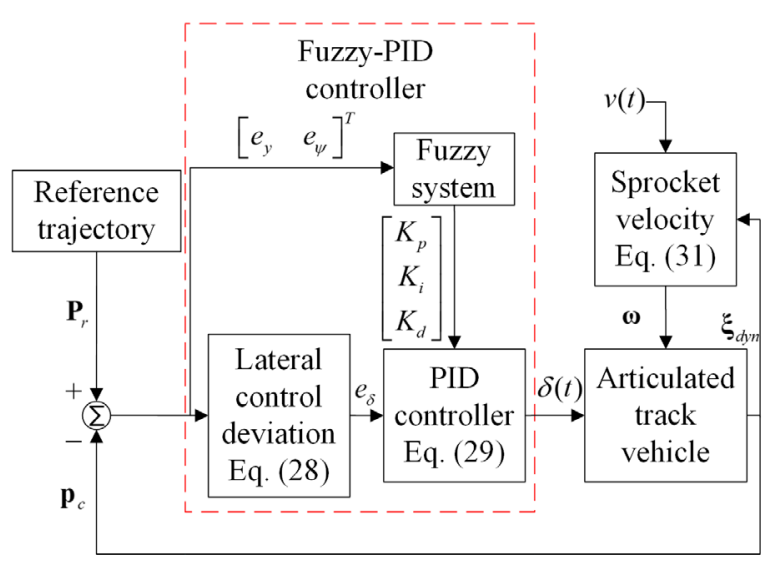

Fig. 5. Model of path-following control for an ATV

The ATV path-tracking control aims to maintain agreement between the position and posture of the vehicle and the pre-set path. The distance and heading angle deviations are as close as possible to zero under the effect of the controller. The complete control 
scheme of the path tracking for ATVs is illustrated in Fig. 5.

\subsection{Sprocket Velocity Control}

According to the kinematics and dynamics analysis, the ATV driven independently by each tracked motor has no differential device. During the steering process, if the sprocket on both sides of the vehicle maintains the same velocity, then the winding velocity of the outer track, relative to the body of the vehicle, will be less than the actual speed of the track. The force generated between the track and the ground will be opposite to the forward direction, which not only reduces the driving efficiency but also makes the actual steering radius of the vehicle substantially larger than the theoretical steering radius and increases the wear on the vehicle. Therefore, we propose an adaptive control of the velocity of the sprocket. According to Eqs. (4) and (8), to generate the same forward traction force on both sides of the track during steering, the velocity of the sprocket on both sides of the track should be expressed as follows:

$$
\begin{aligned}
\omega_{L} & =\frac{1}{r}[\dot{x}+(b / 2) \dot{\psi}], \\
\omega_{R} & =\frac{1}{r}[\dot{x}-(b / 2) \dot{\psi}], \\
\omega_{L}{ }^{\prime} & =\frac{1}{r}\left[\dot{x}^{\prime}+(b / 2) \dot{\psi}^{\prime}\right], \\
\omega_{R}{ }^{\prime} & =\frac{1}{r}\left[\dot{x}^{\prime}-(b / 2) \dot{\psi}^{\prime}\right] .
\end{aligned}
$$

\subsection{Fuzzy Rule}

The fuzzy PID controller of the articulated tracked vehicle has two input variables and three output variables. The input variables are the distance deviation $e_{y}$ and heading angle deviation $e_{\psi}$. The output variables are the control parameters $K_{p}, K_{i}$, and $K_{d}$ of the PID controller. The position and attitude adjustment of the articulated tracked vehicle is based on the deflection angle $\delta$ of the articulated joint.

After adjusting to the appropriate position and attitude, an adjustment within a smaller range is performed to improve the accuracy of the vehicle path tracking. When the variation is high, the distance deviation should be reduced immediately. After adjusting to the appropriate position and attitude, the control range should be reduced to improve the accuracy of the vehicle path tracking. Therefore, setting the fuzzy field of the distance and the heading angle deviations of the articulated tracked vehicle is necessary. The fuzzy field for establishing the range variation is $[-6 \mathrm{~m}, 6 \mathrm{~m}]$, and that of the heading angle deviation is $\left[-30^{\circ}, 30^{\circ}\right]$. The two input variables are divided into five grades: NB (negative large), NM (negative medium), Z (zero), PM (positive middle), and PB (positive large). When the distance and heading angle deviations exceed the corresponding adjustment range, the speed difference between the two sides of the ATV and articulated point deflection angle will be matched to the minimum turning radius to make the vehicle run to the pre-set path as soon as possible. The membership function of distance and heading angle deviations are shown in Fig. 6.

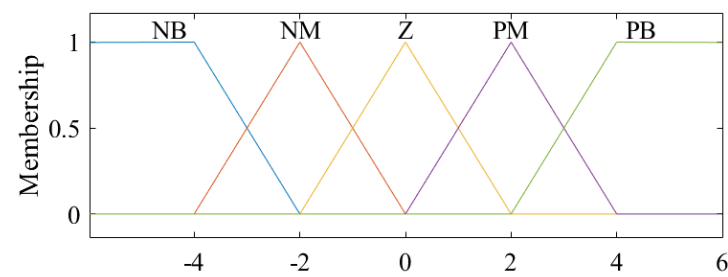

a)

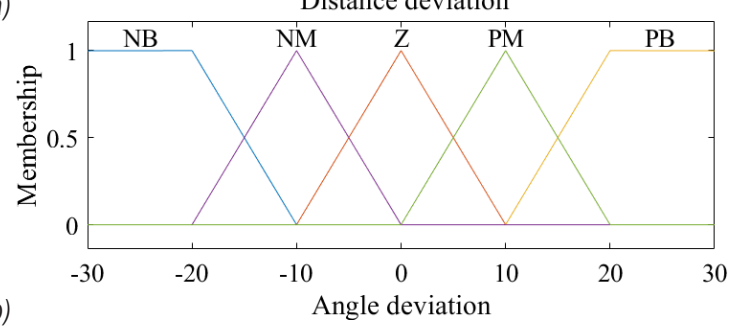

Fig. 6. Membership function of distance deviation and heading angle deviation

A reasonable range for the output variables $K_{p}$, $K_{i}$, and $K_{d}$ of the fuzzy PID controller is selected by parameter testing to achieve the accuracy requirement for the control of the ATV. Increasing the proportional coefficient $\mathrm{Kp}$ in the PID controller can accelerate the response speed and improve the regulation accuracy of the system. The membership adjustment range of $K_{p}$ is divided into five grades: $K_{p 1}, K_{p 2}, K_{p 3}, K_{p 4}$ and $K_{p 5}$ within the range $[1.3,1.7]$. The integral coefficient $K_{i}$ can eliminate the system residual error, and its membership adjustment range is [0.1, 0.15], which is divided into five grades: $K_{i 1}, K_{i 2}, K_{i 3}, K_{i 4}$ and $K_{i 5}$. The derivative coefficient $K_{d}$ can improve the dynamic performance of the system, and its membership adjustment range is [0.01, 0.015], which is divided into five grades: $K_{d 1}, K_{d 2}, K_{d 3}, K_{d 4}$ and $K_{d 5}$. By adjusting and grading the three parameters according to this range, the rapid response and high control precision of the ATV control system can be achieved. 
Table 1. Fuzzy control rule

\begin{tabular}{|c|c|c|c|c|c|c|}
\hline & & \multicolumn{5}{|c|}{$e_{y}$} \\
\hline & & NB & NM & Z & PM & PB \\
\hline \multirow{5}{*}{$e_{\psi}$} & NB & $K_{p 5} / K_{i 1} / K_{d 5}$ & $K_{p 5} / K_{i 1} / K_{d 3}$ & $K_{p 5} / K_{i 1} / K_{d 1}$ & $K_{p 3} / K_{i 2} / K_{d 3}$ & $K_{p 2} / K_{i 2} / K_{d 5}$ \\
\hline & NM & $K_{p 4} / K_{i 1} / K_{d 5}$ & $K_{p 5} / K_{i 2} / K_{d 4}$ & $K_{p 4} / K_{i 2} / K_{d 2}$ & $K_{p 2} / K_{i 3} / K_{d 4}$ & $K_{p 1} / K_{i 4} / K_{d 5}$ \\
\hline & Z & $K_{p 1} / K_{i 4} / K_{d 1}$ & $K_{p 1} / K_{i 5} / K_{d 1}$ & $K_{p 2} / K_{i 5} / K_{d 1}$ & $K_{p 1} / K_{i 5} / K_{d 1}$ & $K_{p 1} / K_{i 4} / K_{d 1}$ \\
\hline & PM & $K_{p 3} / K_{i 4} / K_{d 5}$ & $K_{p 2} / K_{i 3} / K_{d 4}$ & $K_{p 4} / K_{i 2} / K_{d 2}$ & $K_{p 4} / K_{i 2} / K_{d 4}$ & $K_{p 5} / K_{i 1} / K_{d 5}$ \\
\hline & $\mathrm{PB}$ & $K_{p 2} / K_{i 2} / K_{d 5}$ & $K_{p 3} / K_{i 2} / K_{d 3}$ & $K_{p 5} / K_{i 1} / K_{d 1}$ & $K_{p 5} / K_{i 1} / K_{d 4}$ & $K_{p 5} / K_{i 1} / K_{d 5}$ \\
\hline
\end{tabular}

The corresponding membership function is shown in Fig. 7.

The control method for the ATV is expressed as follows:

(1) When the deviation of the distance and heading angle is high, $K_{p}$ assumes a higher value for a fast response. With a decrease in the total deviation, the increase in $K_{p}$ enables the vehicle to maintain the current direction approaching the pre-set path. When the distance deviation of the articulated tracked vehicle is further reduced, the heading angle is the main deviation, and the $K_{p}$ should be higher.

(2) The parameter $K_{i}$ is primarily used to eliminate the residual error caused by the proportional link. Although the parameter can improve the control accuracy and accelerate the response of the system, it can also make the system produce higher oscillation amplitudes. In the process of approaching the pre-set path, $K_{i}$ should be smaller when the deviation of the distance and heading angle is high to avoid excessive oscillation of the system. When the deviation is small, increasing $K_{i}$ can correspondingly improve the accuracy of the system. After passing the marking line, $K_{i}$ is higher to increase the response speed of the system and make the vehicle return to the pre-set path as soon as possible.

(3) Parameter $K_{d}$ has an excellent regulating effect on the dynamic characteristics of the system. When
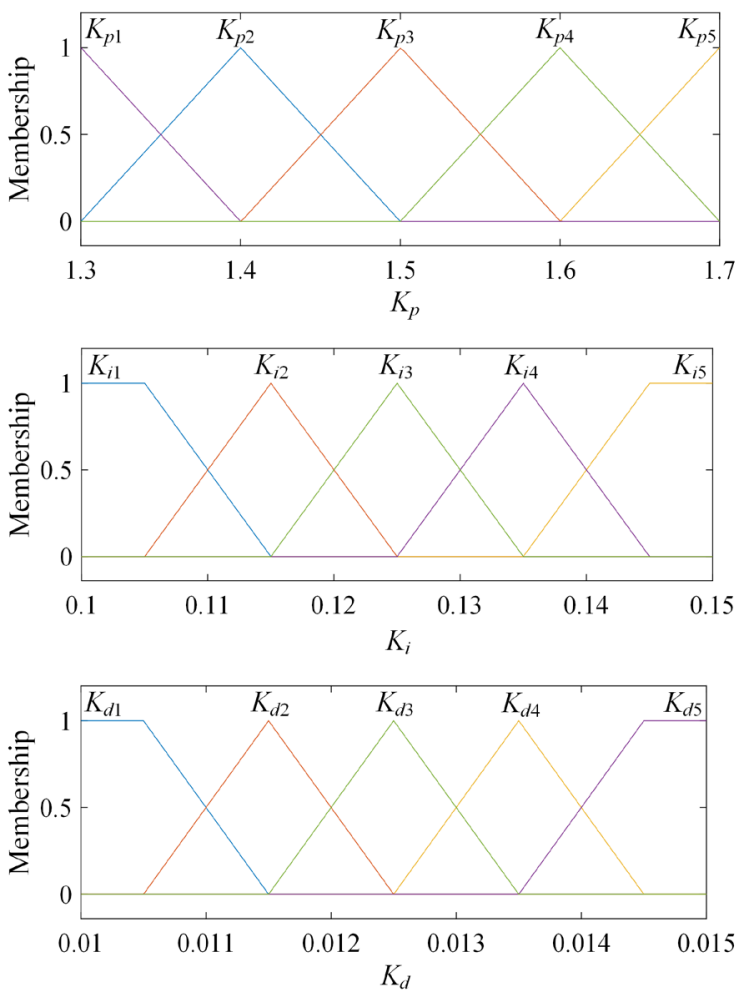

Fig. 7. Membership function of PID controller parameters

the deviation of distance and heading angle is large, $K_{d}$ assumes a higher value to reduce the shock of the system. When the deviation of the
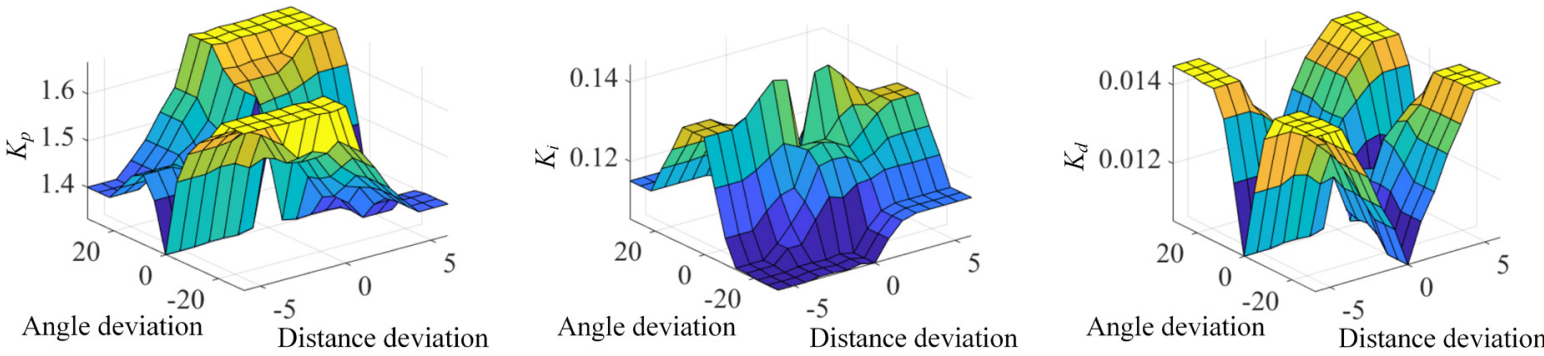

Fig. 8. Relationship between fuzzy control output variables and input variables 


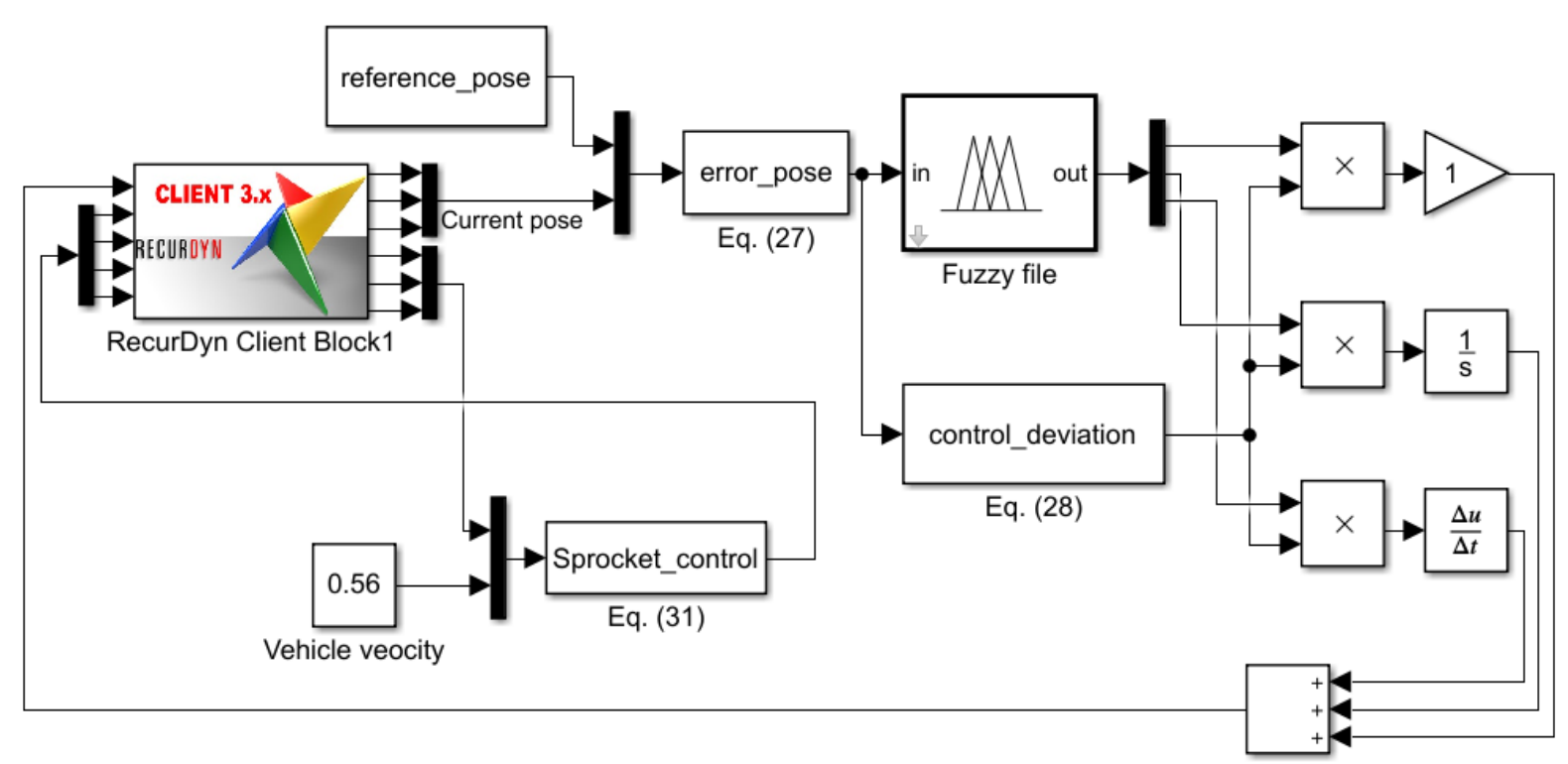

Fig. 9. Control block diagram of the fuzzy PID controller using RecurDyn/Simulink

system is small, the $K_{d}$ value is reduced, to enable the integral link to adjust the error better and to increase the adjusting precision of the system.

The fuzzy rule for the control system, which is designed based on previous work in [20] and [34] and modified through simulation tests, is shown in Table 1. The relationship between the inputs and outputs are shown in Fig. 8.

\section{SIMULATION RESULTS}

To verify the effectiveness of the controller proposed in this paper, a virtual prototype simulation analysis is conducted in two typical driving conditions of the ATV. The accuracy of the vehicle path-tracking control system is determined by analysing the distance and heading angle deviations between the actual path and the pre-set path of the vehicle during driving. The main parameters of the model are shown in Table 2. The Simulink control block diagram of the controller is shown in Fig. 9.

For the straight driving condition, the initial distance and heading angle deviations between the centre of mass of the ATV and the pre-set path are $5.6 \mathrm{~m}$ and $30^{\circ}$, respectively. The driving speed of the centre of mass of the vehicle is $0.56 \mathrm{~m} / \mathrm{s}$. The results of two controllers for the straight driving condition are shown in Fig. 10. Fig. 10a and Fig. 10b illustrate the time-history of the distance deviation and heading angle deviation of the ATV, respectively. Fig. 10c shows the trajectory of the ATV and reference straight path in $X Y$-plane. It can be seen from these figures that the overshoot by the classical PID controller is approximately $26 \%$ and settling time is approximately $110 \mathrm{~s}$, while the overshoot by the fuzzy PID controller is approximately $15 \%$ and the settling time is approximately $90 \mathrm{~s}$. The fuzzy PID shows faster response and less overshoot than the classical PID controller because of the fuzzy system.

Table 2. Design parameters for simulation

\begin{tabular}{|c|c|c|}
\hline & & Values \\
\hline \multirow{6}{*}{$\begin{array}{l}\text { Vehicle } \\
\text { Parameters }\end{array}$} & Ground contact length: $l$ & $1953 \mathrm{~mm}$ \\
\hline & Mass: $m$ & $14.78 t$ \\
\hline & Track gauge: $b$ & $1500 \mathrm{~mm}$ \\
\hline & Track width: $h$ & $600 \mathrm{~mm}$ \\
\hline & Pitch radius of the sprocket: $r$ & $375 \mathrm{~mm}$ \\
\hline & $\begin{array}{l}\text { Distance from the articulation } \\
\text { point to the centre of mass: } d\end{array}$ & $2625 \mathrm{~mm}$ \\
\hline \multirow{6}{*}{$\begin{array}{l}\text { Terrain } \\
\text { Parameters }\end{array}$} & Soil shear deformation modulus: $K$ & $0.02 \mathrm{~m}$ \\
\hline & Apparent cohesion: $c$ & 70000 \\
\hline & Angle of internal shear: $\phi$ & 0.67 \\
\hline & $\begin{array}{l}\text { Friction coefficient between } \\
\text { the tracks and the terrain: } \mu\end{array}$ & 0.9 \\
\hline & Coefficients of lateral motion resistance: $\mu_{t}$ & 0.8 \\
\hline & $\begin{array}{l}\text { Coefficients of longitude } \\
\text { motion resistance: } \mu_{f}\end{array}$ & 0.6 \\
\hline
\end{tabular}

For the curved driving conditions, the pre-set path is an arc with a radius of $25.2 \mathrm{~m}$, and the initial distance and heading angle deviations are both zero, 


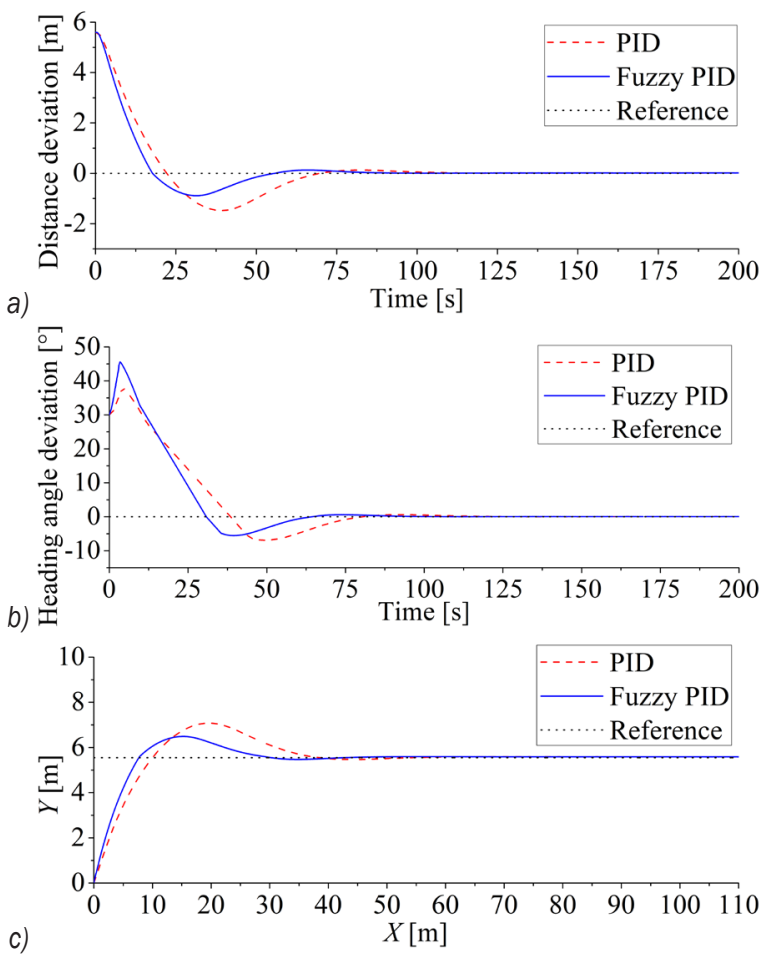

Fig. 10. Simulation results for the two controllers in a straight trajectory; a) distance deviation, b) heading angle deviation, and c) trajectory of the $X Y$-plane

a)

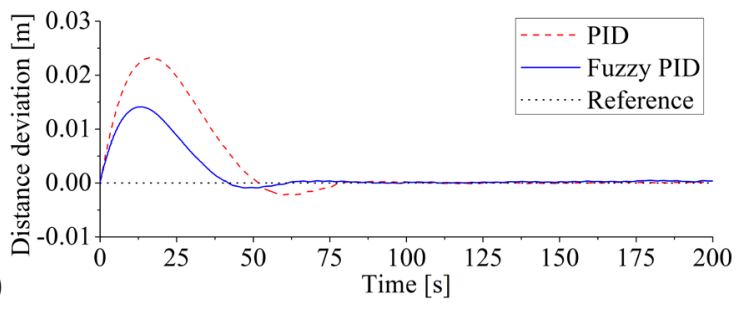

b) $\stackrel{\oplus}{1}$
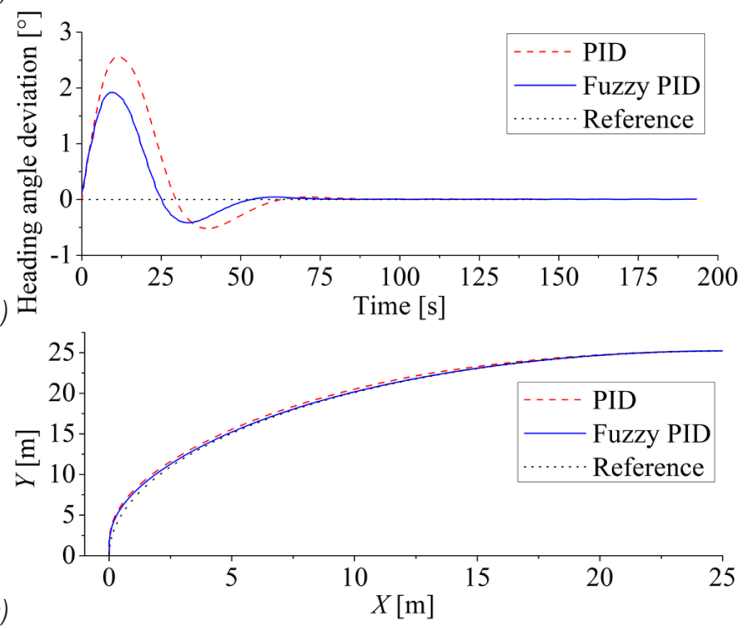

Fig. 11. Simulation results for the two controllers in a circular trajectory; a) distance deviation, b) heading angle deviation, and c) trajectory of the $X Y$-plane and the driving velocity of the vehicle is $0.56 \mathrm{~m} / \mathrm{s}$. Fig. $11 \mathrm{a}$ and Fig. 11b show the time-history of the distance deviation and heading angle deviation and Fig. 11c shows the trajectory of the ATV. From Fig. 11, it can be understood that by using the fuzzy system for tuning the PID gains improves performances.

\section{EXPERIMENTAL VERIFICATION}

The control effect of the ATV path-tracking control system is experimentally investigated to verify the fuzzy PID control algorithm. The overall scheme of the ATV path tracking control test platform is shown in Fig. 12. The test platform is composed of the ATV test prototype, data acquisition and processing scheme, and computer control system. The latter is mainly employed to run the path tracking control system based on visual navigation and collect and analyse relevant data.

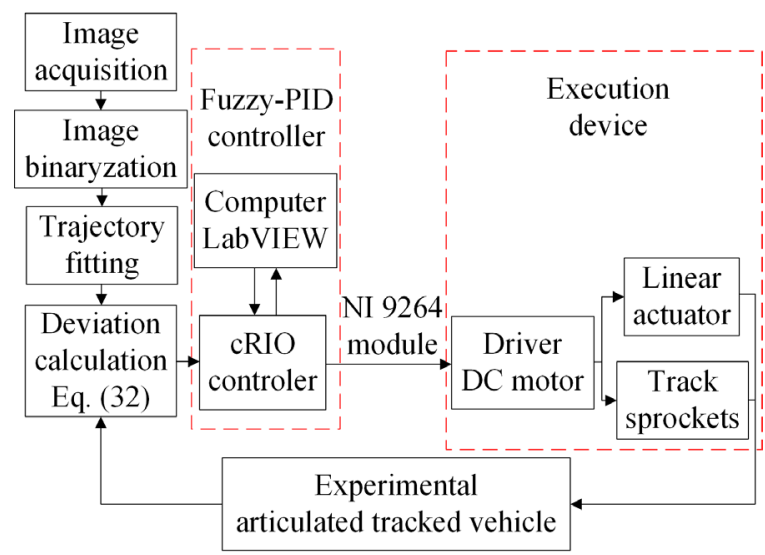

Fig. 12. General scheme of the path tracking control test platform

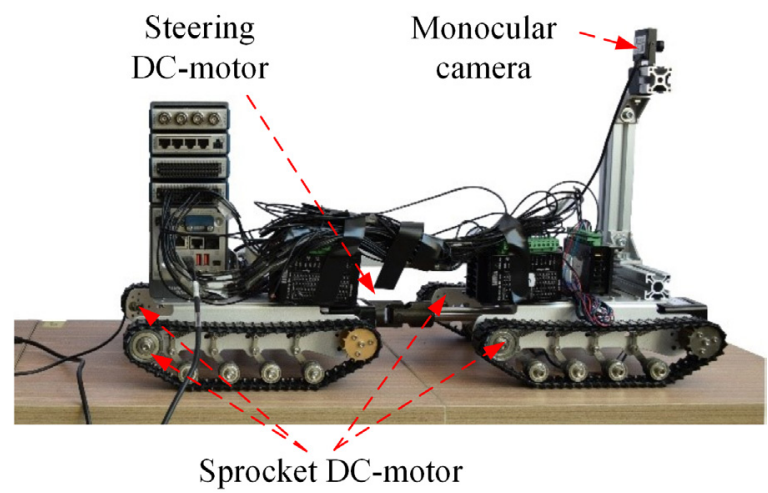

Fig. 13. Overview of the path-tracking control test platform

In the experiment, the pre-set path is set on the horizontal ground. A reduced scale model was employed for the experimental prototype with a ratio of $14: 1$. Since the purpose of the experiment is to 
verify the feasibility of the control algorithm based on visual navigation, the adoption of a small-sized model is acceptable. The data acquisition and processing scheme are input into the computer control system to obtain the running state of the test prototype in the process. The fuzzy PID controller provides the control commands to the actuator of the ATV to follow the pre-set path. The path tracking control platform based on visual navigation is shown in Fig. 13.

a)

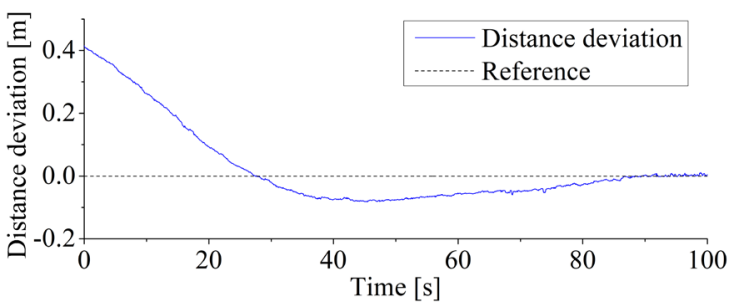

b)

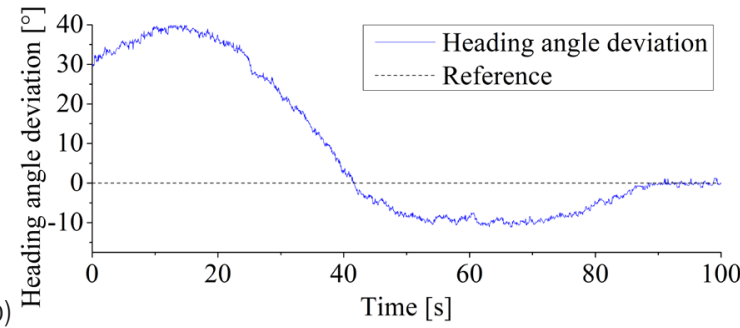

C)
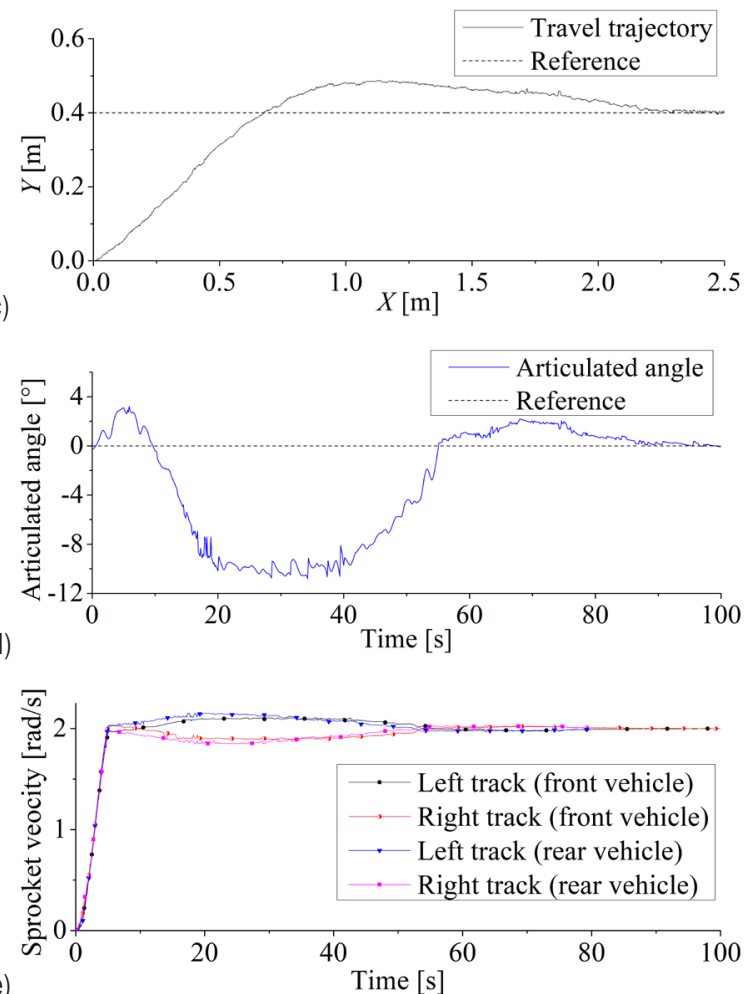

Fig. 14. Experimental results from a straight path; a) distance deviation, b) heading angle deviation, c) trajectory of the XY-plane, d) articulated angle of rotation and e) velocities of sprockets
A monocular camera is used to collect road information in front of the test prototype. The collected image is processed using binarization to identify clear landmark information. A matrix is created by extracting the values of each pixel in the binary image. The sudden change in pixel values is determined and recorded from the starting position to obtain the centreline coordinates of the navigation line. The least-squares method is used to fit the centreline coordinates, and the fitting curve of the road navigation line is obtained. According to the similarity principle and the triangle relationship, the lateral deviation $e_{y}$ and heading angle deviation $e_{\psi}$ can be calculated.

According to the road navigation information collected by the visual navigation path-tracking system and by using the designed fuzzy PID controller, the test prototype is automatically controlled to run along the pre-set path. The distance and heading angle deviations between the actual running and the pre-set path and the track speed on both sides of the prototype are analysed in straight and curve driving conditions.

In the straight travelling condition, the initial distance and heading angle deviations between the centre of mass and the path of the test prototype are $0.4 \mathrm{~m}$ and $30^{\circ}$, respectively. The speed of the driving track is set to $0.1 \mathrm{~m} / \mathrm{s}$. Fig. $14 \mathrm{a}$ and $14 \mathrm{~b}$ illustrate the variation in the distance and heading angle deviations with time. Fig. $14 \mathrm{c}$ shows the trajectory in the $X Y$ plane. The control inputs are shown in Fig. $14 \mathrm{~d}$ and $14 \mathrm{e}$.

In the case of driving along a curved path, the pre-set path is a curve with a $2.5 \mathrm{~m}$ radius. The initial distance and heading angle deviations between the centre of mass and the pre-set path are $0.4 \mathrm{~m}$ and $30^{\circ}$, respectively. Fig. $15 \mathrm{a}$ and $15 \mathrm{~b}$ show the variation in the distance and heading angle deviations with time. The $X Y$-plane motion is shown in Fig. 15c. The corresponding control inputs are demonstrated in Fig. $15 \mathrm{~d}$ and $15 \mathrm{e}$.

According to the tests and analysis, the control system can effectively realize the path tracking of the ATVs by adjusting the sprocket velocity of both sides and the deflection angle of the articulated points in accordance with the different driving paths.

\section{CONCLUSIONS}

A control system for path-tracking for an ATV based on the fuzzy PID algorithm is proposed in this study. The control system uses the distance and heading angle deviations between the actual trajectory and the pre-set path as inputs, and the outputs are the 
a)

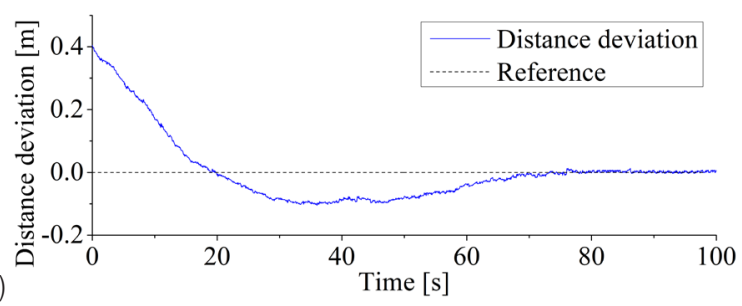

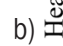

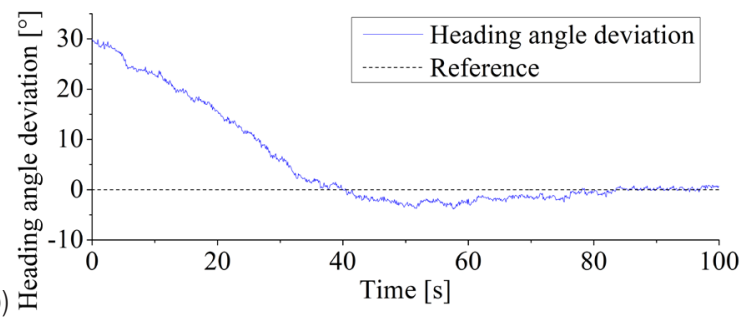

C)
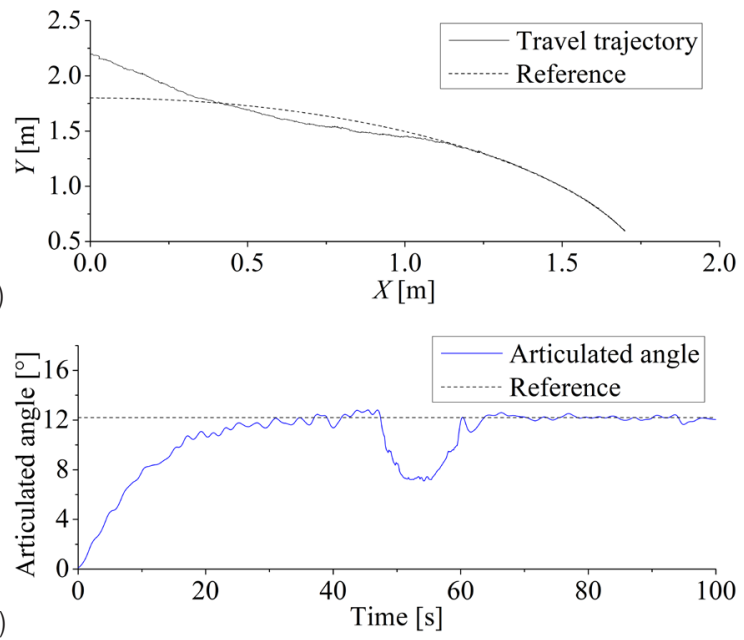

d)

e)

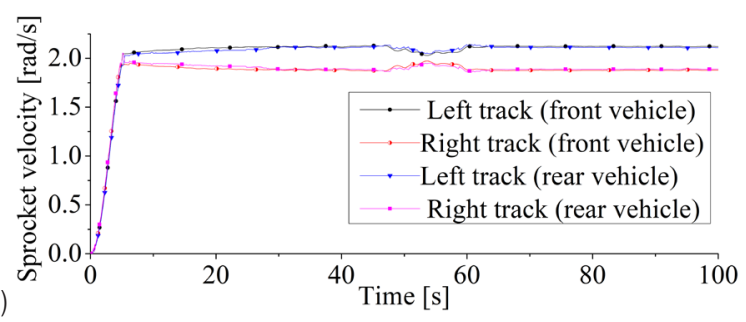

Fig. 15. Experimental results from a circular path; a) distance deviation, b) heading angle deviation, c) trajectory of the XY-plane, d) articulated angle of rotation and e) velocities of sprockets

deflection angle and track velocity on both sides of the ATV. The fuzzy PID controller is the core of the control system, which adjusts the parameters $\mathrm{Kp}, \mathrm{Ki}$, and $\mathrm{Kd}$ according to the prescribed fuzzy rules to achieve effective vehicle tracking. The mathematical model of the ATV is proposed, and a virtual prototype model of the ATV is established in RecurDyn. The validity of the virtual prototype model is verified by a simulation comparison between the prototype and mathematical models. A fuzzy PID control system model is established in Simulink, and the virtual prototype co-simulation analysis is conducted for two typical working conditions, i.e., straight and curve path travelling. The simulation results show that the ATV can effectively track the pre-set path under the control of the fuzzy PID controller.

An ATV path tracking control test platform that is based on visual navigation was developed to test the effects of the control system in a practical application. The vision navigation system is used to collect path tracking information. The fuzzy PID controller is applied for real-time control of vehicle tracking. The distance and heading angle deviation variations during tracking are analysed using the path tracking test for straight and curved driving paths, and the deflection angle and track speed on both sides of the articulated track are adjusted. The experimental results show that the ATV tracking control system is effective in practice.

\section{ACKNOWLEDGMENTS}

This work was supported by the National Natural Science Foundation of China (Grant No. 51775225)

\section{NOMENCLATURES}

$\dot{x}, \dot{y} \quad$ centroid point velocity component of front vehicle, $[\mathrm{m} / \mathrm{s}]$

$\dot{x}^{\prime}, \dot{y}^{\prime}$ centroid point velocity component of front vehicle, $[\mathrm{m} / \mathrm{s}]$

$\psi, \psi^{\prime} \quad$ yaw angle, [rad]

$\delta$ deflection angle between the front vehicle and the rear vehicle, [rad]

$\beta, \beta^{\prime} \quad$ side slip angle, [rad]

$X, Y$ centroid coordinates of front vehicle in global coordinate system, [m]

$v, v^{\prime} \quad$ front and rear vehicle centroid velocity, $[\mathrm{m} / \mathrm{s}]$

$M \quad$ mass of the single vehicle, $[\mathrm{kg}]$

$L \quad$ grounding length of the crawler, [m]

$B \quad$ track gauge, $[\mathrm{m}]$

$D$ distance between the centre point and the articulated point, [m]

$H \quad$ width of link pad, [m]

$I_{Z}$ the moment of inertia of the single vehicle, $\left[\mathrm{kg} \cdot \mathrm{m}^{2}\right]$

$i_{L}, i_{R} \quad$ track slips of front vehicle

$i_{L}{ }^{\prime}, i_{R}{ }^{\prime} \quad$ track slips of rear vehicle

$\omega_{L}, \omega_{R}$ sprocket velocity of front vehicle, $[\mathrm{rad} / \mathrm{s}]$

$\omega_{L}{ }^{\prime}, \omega_{R}{ }^{\prime}$ sprocket velocity of front vehicle, $[\mathrm{rad} / \mathrm{s}]$

$v_{L}, v_{R}$ actual velocity of the crawlers of front vehicle, $[\mathrm{m} / \mathrm{s}]$ 
$v_{L}{ }^{\prime}, v_{R}{ }^{\prime}$ actual velocity of the crawlers of rear vehicle, $[\mathrm{m} / \mathrm{s}]$

$F_{L}, F_{R}$ tractive force of the crawlers of the front vehicle, $[\mathrm{N}]$

$F_{L}^{\prime}, F_{R}{ }^{\prime}$ tractive force of the crawlers of the rear vehicle, $[\mathrm{N}]$

$R_{L}, R_{R}$ longitudinal resistance of the front vehicle, [N]

$R_{L}{ }^{\prime}, R_{R}{ }^{\prime}$ longitudinal resistance of the rear vehicle, [N]

$F_{t x}, F_{t y}$ force component between front vehicle and articulated point, $[\mathrm{N}]$

$F_{t x}{ }^{\prime}, F_{t y}{ }^{\prime}$ force component between rear vehicle and articulated point, [N]

$M \quad$ drag moment of centroid point in front vehicle, $[\mathrm{N} \cdot \mathrm{m}]$

$M^{\prime} \quad$ drag moment of centroid point in rear vehicle, $[\mathrm{N} \cdot \mathrm{m}]$

$F_{\text {max }} \quad$ maximum tractive force produced by a track, $[\mathrm{N}]$

$\tau_{\max } \quad$ maximum shear strength of the terrain, $\left[\mathrm{N} / \mathrm{m}^{2}\right]$

c apparent cohesion, [N/m²]

$\phi \quad$ angle of internal shearing resistance of the terrain, $\left[\mathrm{N} / \mathrm{m}^{2}\right]$

$p \quad$ normal pressure, $[\mathrm{kPa}]$

$K \quad$ soil shear deformation modulus, [m]

$\mu_{f} \quad$ coefficient of longitudinal motion resistance, $[-]$

$\mu_{T} \quad$ coefficients of lateral motion resistance, [-]

$f_{y}, f_{y}{ }^{\prime} \quad$ lateral resistance force of the front vehicle and rear vehicle respectively, [N]

$s_{0}, s_{0}{ }^{\prime} \quad$ longitudinal slip of track velocity instantaneous centre, $[\mathrm{m}]$

$M_{l}, M_{l}^{\prime}$ turning moment produced by longitudinal forces, $[\mathrm{N} \cdot \mathrm{m}]$

$M_{r}, M_{r}{ }^{\prime}$ turning moment produced by lateral forces, $[\mathrm{N} \cdot \mathrm{m}]$

$X_{r}, Y_{r}, \psi_{r}$ components of reference pose, [-]

$e_{x}, e_{y}, e_{\psi}$ deviation components between current pose and reference pose, [-]

$K_{p} \quad$ proportional gain, [-]

$K_{i} \quad$ integral gain, [-]

$K_{d} \quad$ derivative gain, [-]

\section{REFERENCES}

[1] Watanabe, K., Kitano, M., Fugishima, A. (1995). Handling and stability performance of four-track steering vehicles. Journal of Terramechanics, vol. 32, no. 6, p. 285-302, DOI: 285-302, DOI:10.1016/0022-4898(95)00022-4.

[2] Zongwei, Y., Guoqiang, W., Rui, G., Xuefei, L. (2013). Theory and experimental research on six-track steering vehicles.
Vehicle System Dynamics. International Journal of Vehicle Mechanics and Mobility, vol. 51, no. 2, p. 218-235, D0l:10.10 80/00423114.2012.722647.

[3] Nuttall Jr., C.J. (1964). Some notes on the steering of tracked vehicles by articulation. Journal of Terramechanics, vol. 1, no. 1, p. 38-74, D0l:10.1016/0022-4898(64)90123-5.

[4] Zhao, H., Wang, G., Wang, S., Yang, R., Tian, H., Bi, Q. (2018). The virtual prototype model simulation on the steady-state machine performance. Intelligent Automation and Soft Computing, vol. 24, no. 3, p. 581-592, DOI:10.31209/2018.100000025.

[5] Watanabe, K., Kitano, M. (1986). Study on steerability of articulated tracked vehicles-part 1. Theoretical and experimental analysis. Journal of Terramechanics, vol. 23, no. 2, p. 69-83, DOI:10.1016/0022-4898(86)90015-7.

[6] Alhimdani, F.F. (1982). Steering analysis of articulated tracked vehicles. Journal of Terramechanics, vol. 19, no. 3, p. 195209, DOI:10.1016/0022-4898(82)90004-0.

[7] Sasaki, S., Yamada, T., Miyata, E. (1991). Articulated tracked vehicle with four degrees of freedom. Journal of Terramechanics, vol. 28, no. 2-3, p. 189-199, DOI:10.1016/0022-4898(91)90033-3.

[8] Fijalkowski, B.T. (2003). Novel mobility and steerability enhancing concept of all-electric intelligent articulated tracked vehicles. IEEE IV Intelligent Vehicles Symposium. Proceedings, p. 225-230, DOI:10.1109/IVS.2003.1212913.

[9] Wu, J., Wang, G., Zhao, H., \& Sun, K. (2019). Study on electromechanical performance of steering of the electric articulated tracked vehicles. Journal of Mechanical Science and Technology, vol. 33, no. 7, p. 3171-3185, D0l:10.1007/ s12206-019-0612-7.

[10] Karabegović, I., Karabegović, E., Mahmić, M., Husak, E. (2015). The application of service robots for logistics in manufacturing processes. Advances in Production Engineering \& Management, vol. 10, no. 4, p. 185-194, Dol:10.14743/ apem2015.4.201.

[11] Muthukumaran, S., Sivaramakrishnan, R. (2019). Optimal path planning for an autonomous mobile robot using dragonfly algorithm. International Journal of Simulation Modelling, vol. 18, no. 3, p. 397-407, D0l:10.2507/IJSIMM18(3)474.

[12] Kadir, Z.A., Mazlan, S.A., Zamzuri, H., Hudha, K., Amer, N.H. (2015). Adaptive fuzzy-PI control for active front steering system of armoured vehicles: Outer loop control design for firing on the move system. Strojniški vestnik - Journal of Mechanical Engineering, vol. 61, no. 3, p. 187-195, DOl:10.5545/sv-jme.2014.2210.

[13] Popovic, V., Vasic, B., Petrovic, M., Mitic, S. (2011). System approach to vehicle suspension system control in CAE environment. Strojniški vestnik - Journal of Mechanical Engineering, vol. 57, no. 2, p. 100-109, D0l:10.5545/svjme.2009.018.

[14] Zhao, P.X., Luo, W.H., Han, X. (2019). Time-dependent and bi-objective vehicle routing problem with time windows. Advances in Production Engineering \& Management, vol. 14, no. 2, p. 201-212, DOI:10.14743/apem2019.2.322.

[15] Martin, T.C., Orchard, M.E., Sánchez, P.V. (2013). Design and simulation of control strategies for trajectory tracking in an autonomous ground vehicle. IFAC Proceedings Volumes, 
vol. 46 , no. 24 , p. 118-123, DOl:10.3182/20130911-3BR-3021.00096.

[16] Haddad, A., Aitouche, A., Cocquempot, V. (2015). Active fault tolerant decentralized control strategy for an autonomous 2WS4WD electrical vehicle path tracking. IFAC-PapersOnLine, vol. 48, no. 21, p. 492-498, D0l:10.1016/j.ifacol.2015.09.574.

[17] Bascetta, L., Cucci, D.A., Matteucci, M. (2016). Kinematic trajectory tracking controller for an all-terrain Ackermann steering vehicle. IFAC-PapersOnLine, vol. 49, no. 15, p. 13-18, DOI: 10.1016/j.ifacol.2016.07.600.

[18] Li, X., Sun, Z., Cao, D., Liu, D., He, H. (2017). Development of a new integrated local trajectory planning and tracking control framework for autonomous ground vehicles. Mechanical Systems and Signal Processing, vol. 87, p. 118-137, DOI:10.1016/J.ymssp.2015.10.021.

[19] Hong, S., Choi, J.-S., Kim, H.-W., Won, M.-C., Shin, S.-C., Rhee, J.-S., Park, H.-U. (2009). A path tracking control algorithm for underwater mining vehicles. Journal of Mechanical Science and Technology, vol. 23, no. 8, p. 2030-2037, D0l:10.1007/ s12206-009-0436-y.

[20] Wang, S., Zhang, S., Ma, R., Jin, E., Liu, X., Tian, H., Yang, R. (2018). Remote control system based on the Internet and machine vision for tracked vehicles. Journal of Mechanical Science and Technology, vol. 32, no. 3, p. 1317-1331, DOI:10.1007/s12206-018-0236-3.

[21] Zou, T., Angeles, J., Hassani, F. (2018). Dynamic modeling and trajectory tracking control of unmanned tracked vehicles. Robotics and Autonomous Systems, vol. 110, p. 102-111, DOI:10.1016/j.robot.2018.09.008.

[22] Zhou, L., Wang, G., Sun, K., Li, X. (2019). Trajectory tracking study of track vehicles based on model predictive control. Strojniški vestnik - Journal of Mechanical Engineering, vol. 65, no. 6, p. 329-342, D0l:10.5545/sv-jme.2019.5980.

[23] Wang, S., Ge, H., Ma, R., Cui, D., Liu, X., Zhang, S. (2019). Study on the visual tracking control technology of six-crawler machine. Proceedings of the Institution of Mechanical Engineers, Part C: Journal of Mechanical Engineering Science, vol. 233, no. 17, p. 6051-6075, DOI:10.1177/0954406219858168.

[24] Kumar, V., Nakra, B.C., Mittal, A.P. (2011). A review on classical and fuzzy PID controllers. International Journal of Intelligent Control and Systems, vol. 16, no. 3, p. 170-181.

[25] Choi, Y.J., Chung, W.K. (2004). Introduction. Choi, Y.J., Chung, W.K. (eds). PID Trajectory Tracking Control for Mechanical
Systems. Lecture Notes in Control and Information Science, vol. 298, 3-7, D0l:10.1007/978-3-540-40041-7_1.

[26] Morari, M., Lee, J.H. (1999). Model predictive control: past, present and future. Computers \& Chemical Engineering, vol. 23, no. 4-5, p. 667-682, D0I:10.1016/S0098-1354(98)00301. 9.

[27] Nasir, A.N. K.,Ahmad, M.A., Ismail, R.R. (2010). The control of a highly nonlinear two-wheels balancing robot: A comparative assessment between LQR and PID-PID control schemes. World Academy of Science, Engineering and Technology, vol. 70, p. 227-232.

[28] Carlucho, I., Menna, B., De Paula, M., Acosta, G.G. (2016). Comparison of a PID controller versus a LQG controller for an autonomous underwater vehicle. 3rd IEEE/OES South American International Symposium on Oceanic Engineering $\mathrm{p}$. 1-6. DOI:10.1109/SAISOE.2016.7922475.

[29] Khan, A.A., Rapal, N. (2006). Fuzzy PID controller: design, tuning and comparison with conventional PID controller. IEEE International Conference on Engineering of Intelligent Systems, p. 1-6, D0I:10.1109/ICEIS.2006.1703213.

[30] Kumar, V., Nakra, B.C., Mittal, A.P. (2011). A review on classical and fuzzy PID controllers. International Journal of Intelligent Control and Systems, vol. 16, no. 3, p. 170-181.

[31] Bekker, M.G. (1956). Theory of Land Locomotion: the Mechanics of Vehicle Mobility. Ann Arbor: The University of Michigan Press.

[32] Wong, J.Y. (2008). Theory of Ground Vehicles. John Wiley \& Sons, New York.

[33] Ang, K.H., Chong, G., Li, Y. (2005). PID control system analysis, design, and technology. IEEE Transactions on Control Systems Technology, vol. 13, no. 4, p. 559-576, D0l:10.1109/ TCST.2005.847331.

[34] Tiep, D.K., Lee, K., Im, D.-Y., Kwak, B., Ryoo, Y.-J. (2018), Design of fuzzy-PID controller for path tracking of mobile robot with differential drive. International Journal of Fuzzy Logic and Intelligent Systems, vol. 18, no. 3, p. 220-228, D0l:10.5391/ IJFIS.2018.18.3.220.

[35] Wu, H., Su, W., Liu, Z. (2014). PID controllers: Design and tuning methods. 9th IEEE Conference on Industrial Electronics and Applications, p. 808-813, D0l:10.1109/ ICIEA.2014.6931273.

[36] Li, H.-X. (1997). A comparative design and tuning for conventional fuzzy control. IEEE Transactions on Systems, Man, and Cybernetics, Part B (Cybernetics), vol. 27, no. 5, p. 884-889, D0I:10.1109/3477.623242. 